\title{
EFECTO DE LA LUZ EN EL CRECIMIENTO DE PLANTULAS DE Dipteryx micrantha Harms "SHIHUAHUACO" TRANSPLANTADAS A SOTOBOSQUE, CLAROS Y PLANTACIONES
}

\section{THE EFFECT OF LIGHT ON THE GROWTH OF SEEDLINGS AND SAPLINGS OF Dipteryx micrantha IN UNDERSTORY, GAPS AND PLANTATIONS}

\author{
Mónica Romo Reátegui ${ }^{1}$
}

\begin{abstract}
Resumen
Dipteryx ha sido mencionado como un género que depende de los claros para su crecimiento, aunque como la mayoría de las especies de árboles de los bosques maduros, ésta es tolerante a bajos niveles de luz en su estadio de plántula. Para explorar el efecto de la luz en el crecimiento durante los estadios iniciales de Dipteryx micrantha Harms se hicieron experimentos de transplante a lugares con diferentes niveles de luz. El crecimiento de plántulas transplantadas luego de un año de edad a claros del bosque, es mayor que el crecimiento de plántulas transplantadas a la misma edad a sotobosque y está relacionado con la luz. Luego de tres a cuatro años, los claros se "cierran" y los niveles de luz en estos regresan a niveles similares a los del sotobosque. El análisis de regresión escalonada mostró que en claros, la luz es el factor más determinante en el crecimiento en diámetro y altura de las plántulas, sin que influya en este crecimiento, ni el diámetro inicial de la planta ni el número inicial de hojas.

A niveles más altos de luz, como los individuos en plantaciones mixtas o puras, estos crecen comparativamente mucho más que aquellos que crecen en claros.

Se observa que la influencia de la luz en el crecimiento depende del nivel de ésta, así existe una relación entre la luz y el crecimiento a niveles de luz observados en los claros. Sin embargo no hay relación en niveles de luz tan bajos como en el sotobosque o en ambientes de mucha luz como en las plantaciones puras.
\end{abstract}

Palabras clave: Dipteryx micrantha, plántulas, crecimiento, claros, plantaciones.

\begin{abstract}
Dipteryx has been mentioned to be a gap dependent genus, however, most of the canopy tropical trees tolerate low light levels in their seedling/sapling stage. To explore the effect of light on growth during the early life of Dipteryx micrantha Harms, transplant experiments to different light levels were done. Growth in one-year-old seedlings transplanted to gaps and understory was higher in gaps, and related to light levels. For gaps, stepwise regression analyses showed that light is the most determinant factor on growth in diameter and height, while initial plant diameter or number of leaves were not influenced.

Individuals in plantations grew much better than individuals in gaps with no notorious difference in their morphology compared to those in the forest.

In general, the influence of light on growth depends on whether light levels are high, intermediate or low: it this study, growth was related to middle light levels such as those in the gaps, but it was not related to low light levels such as those of the understory or, to high light levels such as those in plantations.
\end{abstract}

Key words: Dipteryx micrantha, seedlings, saplings light, growth, gaps, plantations

\section{Introducción}

En el bosque húmedo tropical en general, la luz es uno de los factores que más afecta la supervivencia y crecimiento de las plántulas (también llamadas regeneración natural). Sin embargo, los niveles de luz en el sotobosque donde se encuentran las plántulas son muy bajos, variando entre $0.5-30 \%$ de luz abierta. La mayoría de las veces los niveles de luz se encuentran alrededor del 2\% (Canham, 1989; Chazdon \& Fetcher, 1984; Chazdon, 1988; Clark et al., 1993; Smith et al., 1992; Osunkoya et al., 1994; Zagt, 1997). Sólo en los claros del bosque los niveles de luz pueden llegar a 10-30\% de luz (Canham, 1989; Osunkoya et al., 1993; Osunkoya et al., 1994; Van der Meer, 1998) permitiendo un considerable incremento en el crecimiento de los estadios jóvenes. Sin embargo los claros se cierran o cubren en pocos meses (42-64 meses) (De Steven, 1988; Brokaw \& Whitmore, 1992; Van der Meer, 1998; Fraver et al.,1998) retornando a niveles de luz similares a los del bosque circundante.

No sólo el tiempo de los claros es corto sino también su frecuencia. En Cocha Cashu, el promedio 
de mortalidad de árboles es de $1.6 \%$ (Gentry \& Terborgh, 1990) sugiriendo que las oportunidades para que los árboles (o plántulas) crezcan más que el promedio debido a la luz producida por un claro, son en realidad raras. Así en el sotobosque, un ambiente de muy poca luz, el crecimiento de las plántulas es muy lento con eventos de crecimiento mayor sólo según la frecuencia de caídas de árboles y la existencia de claros de luz. En consecuencia, el crecimiento de un individuo antes de llegar a ser un árbol adulto del dosel es extremadamente lento y puede llegar a cientos de años en muchas especies de árboles (Canhan, 1989), especialmente aquellas de maderas duras. Para el caso de Dipteryx panamensis (Pittier) Record \& Mell. por ejemplo, una especie congenérica y muy similar a Dipteryx micrantha Harms "shihuahuaco", la edad de una plántula de $4 \mathrm{~cm}$ de diámetro se calcula que es de 72 años (Clark \& Clark, 1992). El estudio anteriormente mencionado, que además incluyó otras 5 especies de árboles tropicales, demostró que la existencia de individuos en un bosque tropical es influenciada principalmente por la supervivencia y crecimiento cuando las plantas tienen menos de $4 \mathrm{~cm}$ de diámetro. Es entonces muy importante estudiar y comprender los factores que las afectan durante esta edad, especialmente el factor luz.

En contraste con los ambientes naturales, la luz en ambientes deforestados y plantaciones forestales tiene niveles significativamente mayores. Aunque hay especies que no crecen en plantaciones con la misma morfología o cualidades de la madera que los que crecen en bosques naturales, este no es el caso de Dipteryx, tanto D. panamensis (Lois et al., 2003) como D. odorata (Volpato et al., 1973), puesto que se reportan árboles "normales" en plantaciones.

El objetivo de este estudio es comprender el efecto de la luz sobre el crecimiento de plántulas de Dipteryx micrantha transplantadas a lugares con diferentes niveles de ésta. Específicamente, nos preguntamos: ¿Hay diferencias en los efectos de la luz en el crecimiento de plántulas transplantadas al sotobosque, a claros, a plantaciones mixtas y a plantaciones puras?

\section{Materiales y Métodos \\ Lugar de estudio, transplantes y plantaciones}

Transplante a claros.- El estudio fue realizado en la Estación Biológica de Cocha Cashu, Parque Nacional del Manu, Madre de Dios (S 11 ${ }^{\circ} 55^{\prime}$ y O $77^{\circ} 18^{\prime}, 280$ msnm). Cocha Cashu se encuentra ubicado en la zona climática límite entre el bosque húmedo tropical y subtropical. El lugar es un bosque inundable con una precipitación promedio anual de $2000 \mathrm{~mm}$ y una temperatura de 23 a $24{ }^{\circ} \mathrm{C}$. Normalmente, la lluvia se concentra durante los meses de Noviembre a Mayo, mientras que sólo cae aproximadamente $100 \mathrm{~mm}$ durante la estación seca. Eventos climáticos drásticos que producen gran cantidad de lluvia pueden ocurrir durante algunos años, como "El Niño" de 1997 y 1998. Según análisis de los datos climáticos de la Estación ( www.duke/ manu), se observó que las estaciones secas del año 1998 y 1999 tuvieron dos meses de sequía y no un mes como normalmente sucede otros años. El experimento de transplante fue realizado en el bosque aluvial maduro de Cocha Cashu. Este bosque está entre las áreas más grandes de bosque maduro a lo largo del río Manú y entre los de mayor densidad de Dipteryx (Romo et al., 2004); de tal manera Cocha Cashu ha sido llamado un "bosque de Dipteryx-Quararibea" (Janson \& Emmons, 1990). Una descripción detallada del área se encuentra en Gentry \& Terborgh (1990) y Foster (1990).

Para verificar el desarrollo de Dipteryx micrantha Harms en los claros se realizó un experimento de transplante de plántulas a estos ambientes. Las semillas que dieron origen a estas plantas fueron sembradas en Octubre de 1998 y colocadas dentro de dos jaulas de malla metálica para prevenir la depredación por parte de roedores, además de una malla de plástico ("screen mesh") para prevenir el sol directo permitiendo la entrada de agua. Las jaulas estaban en un ambiente de gran cantidad de luz como es el claro de la Estación Biológica que tiene aproximadamente $20 \mathrm{~m}$ de diámetro. Luego de un año (Octubre 1999) un total de 60 de las plántulas más altas (promedio $422 \mathrm{~mm}$ de altura y $6 \mathrm{~mm}$ diámetro) procedentes de las jaulas fueron transplantadas a un sitio del sotobosque (20 plántulas) y a dos sitios en claros (20 plántulas en cada uno). Las plántulas transplantadas al sotobosque, claro 1 y claro 2 fueron igualmente escogidas entre las más altas y eran de igual tamaño tanto en diámetro como en altura (ANOVA diámetro en $\mathrm{mm}: \mathrm{F}=0.84, \mathrm{P}=0.58$, altura en $\mathrm{mm}: \mathrm{F}=0.18, \mathrm{P}=0.83$ ). El claro 1 estaba situado a 250 $\mathrm{m}$ del borde del lago y medía alrededor de $20 \mathrm{~m}$ de diámetro por lo que puede considerarse un claro mediano (Brown, 1993). El claro 2 estaba cerca al lago y media alrededor de $20 \times 10 \mathrm{~m}$ por lo que puede ser considerado un claro pequeño. Aunque algunos estudios demostraron que existía una variedad de microhábitats dentro de los claros (Brown, 1993; Dalling et al., 1999; Denslow et al., 1998), Cintra \& Horna (1997) no encontraron diferencias en el desarrollo de las plántulas de Dipteryx micrantha Harms recién germinadas y transplantadas a un claro, entre aquellas cercanas al tronco y las de la zona de dosel del árbol caído. En este estudio, las plántulas fueron colocadas en dos líneas paralelas de 10 plántulas cada una separadas por $4 \mathrm{~m}$ entre sí. Las líneas comenzaban en el borde del claro.

Plantaciones puras y mixtas.- El sembrado de las plántulas luego de aproximadamente tres meses de germinadas fue realizado en un terreno en el río Tambopata, Madre de Dios (300 msnm). Tambopata está situado en la zona de bosque húmedo subtropical. La precipitación y temperatura son similares a las de 
Cocha Cashu. La zona de trabajo en Tambopata está localizada en el bosque aluvial, similar al de Cocha Cashu, pero en donde la deforestación y presencia de charcas a lo largo del río son un rasgo general. La plantación pura se realizó en una zona de bajío. El ambiente de bajío estaba situado en $12^{\circ} 39^{\prime} 23.22$ ' ' S y $69^{\circ} 11$ ' 26.56', O y el ambiente de chacra agroforestal aproximadamente a $200 \mathrm{~m}$ del bajío y un poco más lejos del río en $12^{\circ} 39^{\prime} 40.25^{\prime}$ ' S y $69^{\circ} 11^{\text {' }}$ 50.89' O.

La siembra de plántulas recién germinadas en las plantaciones puras en el bajío fue realizada en Diciembre de 1998 y la siembra en el ambiente agroforestal en Diciembre de 1999. En el bajío, donde las plantas fueron sembradas distancias de 5 a $8 \mathrm{~m}$ entre si, un canal se encontraba muy cercano a la zona en que se sembraron las plántulas y éste se inundaba completamente durante gran parte de la época de lluvia (Noviembre a Mayo). Las plántulas en la chacra agroforestal sin embargo no se inundaban tan drásticamente aunque la chacra podía tener charcos de agua si las lluvias eran intensas. La chacra agroforestal contenía además plantas de Musa paradisiaca L. "plátano", Cedrela odorata L. "cedro", Swietenia macrophylla King "caoba", Bertholletia excelsa Humb \& Bonpl. "castaña” y Bactris gasipaes Kunth "pihuajo" entre otras.

\section{Medidas de luz}

Las mediciones de luz fueron hechas con el método de fotos hemisféricas. Para el caso de las plántulas transplantadas a claros, las fotos fueron tomadas en Noviembre de 1999, Noviembre de 2000 y Marzo de 2002. El número de fotos de un año a otro fue reduciéndose debido a la mortalidad de las plántulas. Una plántula en el claro 1 fue destrozada entre el tiempo de transplante y toma de foto reduciendo la muestra a 19 plántulas. La luz en el ambiente de plantaciones puras se puede decir que era total ya que las plantas estaban muy distanciadas entre sí, por lo que se considera estos niveles de luz como máximos o de o más de $50 \%$ de luz. No se tomaron fotos hemisféricas a estos individuos que ya eran pequeños arbolitos de más de $3 \mathrm{~m}$ cuando se midieron. En la chacra agroforestal en cambio las plantas podían estar cubiertas o mezcladas con otras especies por lo que se tomaron fotos hemisféricas en Enero de 2001, es decir dos años luego de ser sembradas.

Las fotos hemisféricas son un método indirecto muy confiable para medir la luz y son particularmente adecuadas para este tipo de estudio. Las fotos fueron tomadas al nivel de las hojas superiores de las plántulas con una cámara Minolta y un lente ojo de pez que permite tener un ángulo de casi $180^{\circ}$ y usando una película blanco y negro de 400 ASA, Tri X Pan Kodak. Los negativos fueron escaneados a un nivel de 256 en la escala de grises. Las imágenes fueron manipuladas de tal forma que los grises de las hojas fueron convertidas a negro y el cielo en blanco, convirtiendo las imágenes en solo dos colores, blanco y negro. El programa Winphot (Hans ter Steege, Utrecht University, www.bio.uu.nl/ herba/Guyana/winphot/wp_index.ht m) fue utilizado para calcular el \% de luz (blanco) del área de las fotos. Nos referimos a estas medidas como niveles de luz.

\section{Medidas de crecimiento}

Las medidas del diámetro y altura de las plántulas transplantadas a claros fueron realizadas al inicio en Noviembre de 1999 y remedidas en Noviembre de 2000 y Marzo de 2002. Estas medidas fueron realizadas sólo una vez (Enero de 2001) para el caso de las plántulas en plantaciones puras y mixtas por lo que este caso sólo expresa el crecimiento desde 1998 a 2001 para el caso de las plántulas en plantaciones puras y desde 1999 a 2001 para el caso de las plántulas en plantaciones mixtas. El diámetro fue medido a $1 \mathrm{~cm}$ del suelo a excepción de los individuos en plantaciones puras que ya eran arbolitos, cuyo diámetro fue medido a $1 \mathrm{~m}$ del suelo aproximadamente, altura a la que el diámetro ya era uniforme. La altura fue medida desde el suelo hasta la base del peciolo de la hoja más joven y el número de hojas contado. Dipteryx tiene hojas compuestas alternas. El diámetro fue medido con calibradores de plástico a $0.1 \mathrm{~mm}$ de aproximación. La altura se determinó con cinta de medir a $1 \mathrm{~mm}$ de aproximación.

\section{Análisis estadístico}

Para el caso del experimento de transplante a claros, se utilizó el Test de Mann-Whitney (no paramétrico) para comparar los niveles de luz recibidos por las plántulas en el sotobosque y claros. El mismo test se usó para comparar el crecimiento en diámetro, altura y cambio en número de hojas de las plántulas en claros entre 1999-2000 y 2000-2002. Aunque en este caso, los intervalos de tiempo entre las medidas fueron diferentes ( 1 año vs. 1 año 4 meses) y se esperaría que a mayor tiempo mayor crecimiento. Se utilizó la regresión escalonada ("stepwise regresión”) para ver cual de los factores (luz, diámetro inicial, número de hojas) es más determinante para el crecimiento. Considerando que el diámetro y altura pueden ser funciones del tamaño se grafico el crecimiento como: $\ln$ medida $t_{2}$ - $\ln$ medida $t_{1}$.

Para los individuos en plantaciones, se describe la relación entre la luz y el tamaño de la planta en diámetro y altura usando una regresión lineal. Se asume que los niveles de luz durante el primer año fueron mayores que los registrados el segundo año (2001). Se describe la relación entre diámetro y altura de los individuos.

\section{Resultados}

Transplante a claros.

Un total de $13(65 \%)$ y 34 (85\%) plántulas transplantadas en 1999 al sotobosque y claros 
respectivamente sobrevivió hasta la última vez que fueron medidos en Marzo de 2002.

Los niveles de luz encontrados en los claros son en general más altos que los encontrados en el sotobosque tanto en el primer año $(\mathrm{P}<0.0001)$ como en el segundo año $(\mathrm{P}<0.0001)$ pero en el tercer año los niveles de luz en los claros han disminuido y no son ya estadísticamente diferentes de los encontrados en el sotobosque (Figura 1). Los niveles de luz recibidos por las plántulas al ser transplantadas tuvieron un rango entre 1.54 y $16.86 \%$ (promedio y DE:7.28+3.30). El valor máximo de niveles de luz encontrado es aparentemente el máximo valor posible ya que una foto tomada en el centro del claro grande a $1.20 \mathrm{~m}$ del suelo dio un nivel de luz de $20 \%$.

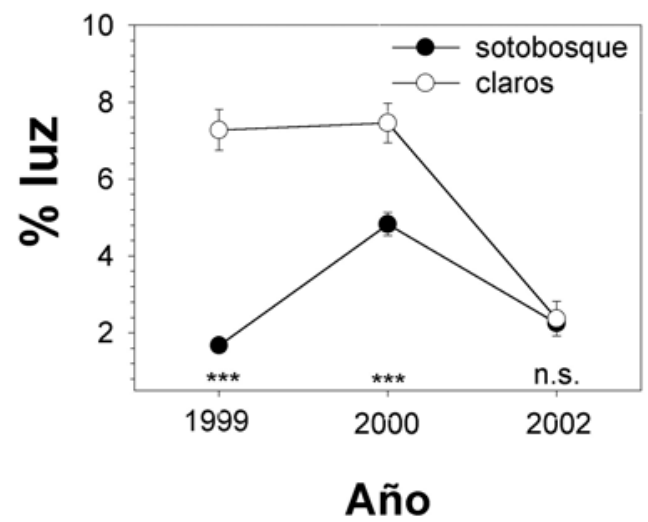

Figura 1. Porcentaje de luz recibido durante tres años por las plántulas de Dipteryx micrantha transplantas a sotobosque y claros. Los símbolos expresan la media y la desviación estándar. $* * *=\mathrm{P}<0.001$, n.s. $=$ no significativamente diferente.

El crecimiento de las plántulas durante los dos años fue mayor en los claros que el sotobosque tanto en diámetro $(\chi+\mathrm{DE}=2.8 \pm 2.3 \mathrm{~mm}$ y $1.9 \pm 2.16 \mathrm{~mm}$ vs. $-0.09 \pm 0.9 \mathrm{~mm}$ y $-0.01 \pm 0.82 \mathrm{~mm})$ como en altura $(\chi+\mathrm{DE}=276+187 \mathrm{~mm}$ y $339 \pm 277 \mathrm{~mm}$ vs. $-4.4+$ $42 \mathrm{~mm}$ y $-6.00 \pm 10 \mathrm{~mm}$ ) (Figura 2). Alrededor de la tercera parte de las plántulas de los claros creció más de $400 \mathrm{~mm}$ por año. En los claros, el crecimiento en diámetro fue ligeramente mayor de 1999 a 2000 que del 2000 a $2002(\mathrm{U}=794, \mathrm{P}=0.0165)$ pero no hubo diferencia en la tasa de crecimiento en altura entre ambos años (Figura 2).

El análisis de regresión escalonada mostró que, en los claros, la luz es el factor mas determinante para el crecimiento en diámetro y altura, no teniendo influencia el diámetro inicial de la planta o el número inicial de hojas (Tabla 1). Los valores de $\mathrm{r}^{2}$ son de $0.26,0.31$ y $0.47(\mathrm{P}<0.01)$ para el crecimiento en diámetro el primer año y en altura el primer y segundo años respectivamente. Ya que la regresión escalonada mostró que la luz es el factor más determinante, se graficó la regresión lineal entre $\%$ de luz $y$ crecimiento en diámetro y altura de las plántulas de claros (Figura 3).

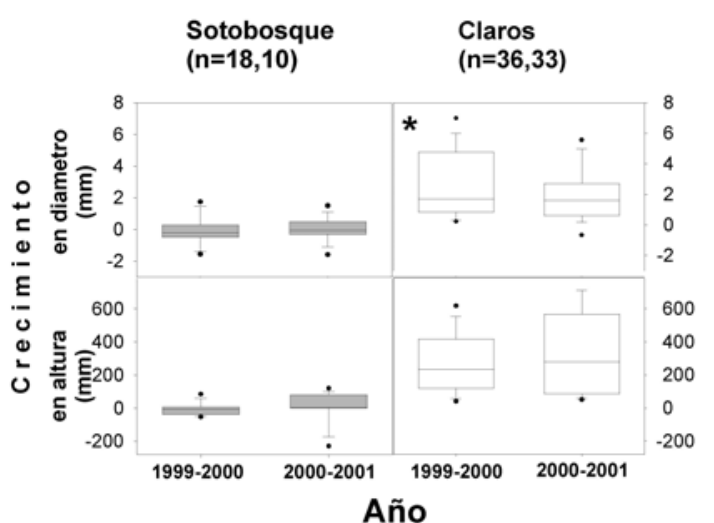

Figura 2. Comparación del crecimiento en diámetro y altura de plántulas transplantadas a sotobosque y a claros. Comparación del crecimiento en diámetro y en altura en dos años consecutivos de plantas transplantadas a sotobosque y claros. Las barras muestran el promedio y el percentil en $95 \% *=\mathrm{P}<0.05$

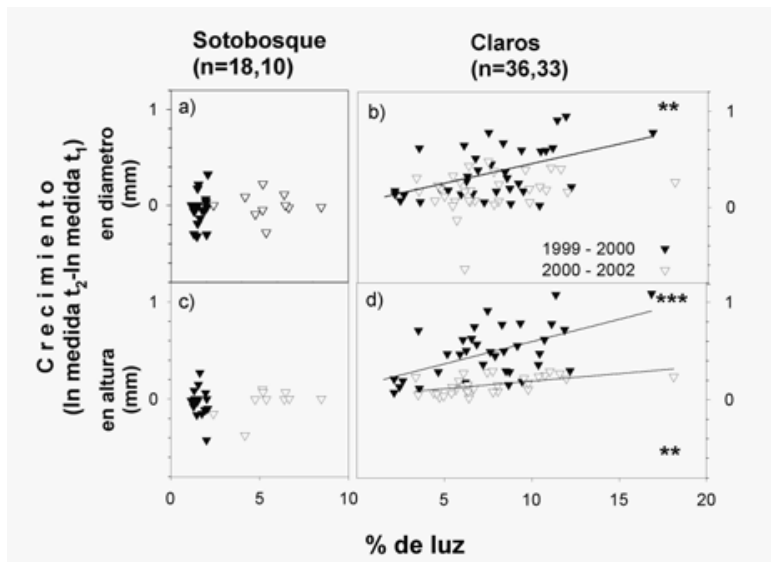

Figura 3. Regresión lineal entre el \% luz y el crecimiento en diámetro y altura en plántulas transplantadas al sotobosque y claros. Ecuación de la regresión linear de b) $\mathrm{r}^{2}=0.26 \mathrm{y}=0.03804+0.04488 \mathrm{x}$ d) $\mathrm{r}^{2}=0.31, \quad \mathrm{y}=0.1385+0.0458 \mathrm{x} \quad$ y $\mathrm{r}^{2}=0.29$, $\mathrm{y}=0.0087+0.0154 \mathrm{x}$, El crecimiento es expresado como: (ln diámetro $t_{2}$-ln diámetro $t_{1}$ ) y $\left(\ln\right.$ altura $t_{2}-\ln$ altura $\left.\mathrm{t}_{1}\right) . \quad *=\mathrm{P}<0.05, * * \mathrm{P}=<0.01, * * *=\mathrm{P}<0.001$.

Para las plántulas transplantadas al sotobosque, ni los niveles de luz, ni el diámetro inicial, ni el número de hojas mostró influencia en el crecimiento.

Plantaciones puras y mixtas.

No sólo el nivel de luz es alto en las plantaciones puras sino también en las mixtas $(\chi+\mathrm{DE}: 25 \pm 9 \%, \mathrm{n}=30$, rango $9-52 \%$ ) por lo que se sugiere que la luz no fue un factor limitante en ninguno de estos ambientes. La regresión lineal muestra que no hay relación entre el diámetro y la luz $\left(\mathrm{r}^{2}=0.00, \mathrm{P}=0.67\right)$ o entre la altura $\mathrm{y}$ la luz de las plantas de la plantación mixta $\left(\mathrm{r}^{2}=0.002\right.$, 
Tabla 1. Regresión escalonada del efecto de la luz, diámetro inicial y número de hojas en el crecimiento en diámetro, altura y cambio en número de hojas. El crecimiento es expresado como: $\ln$ medida $t_{2}-\ln$ medida $t_{1}$ $\mathrm{ns}=$ no significativo estadísticamente $*=\mathrm{P}<0.05, * * \mathrm{P}=<0.01, * * *=\mathrm{P}<0.001$.

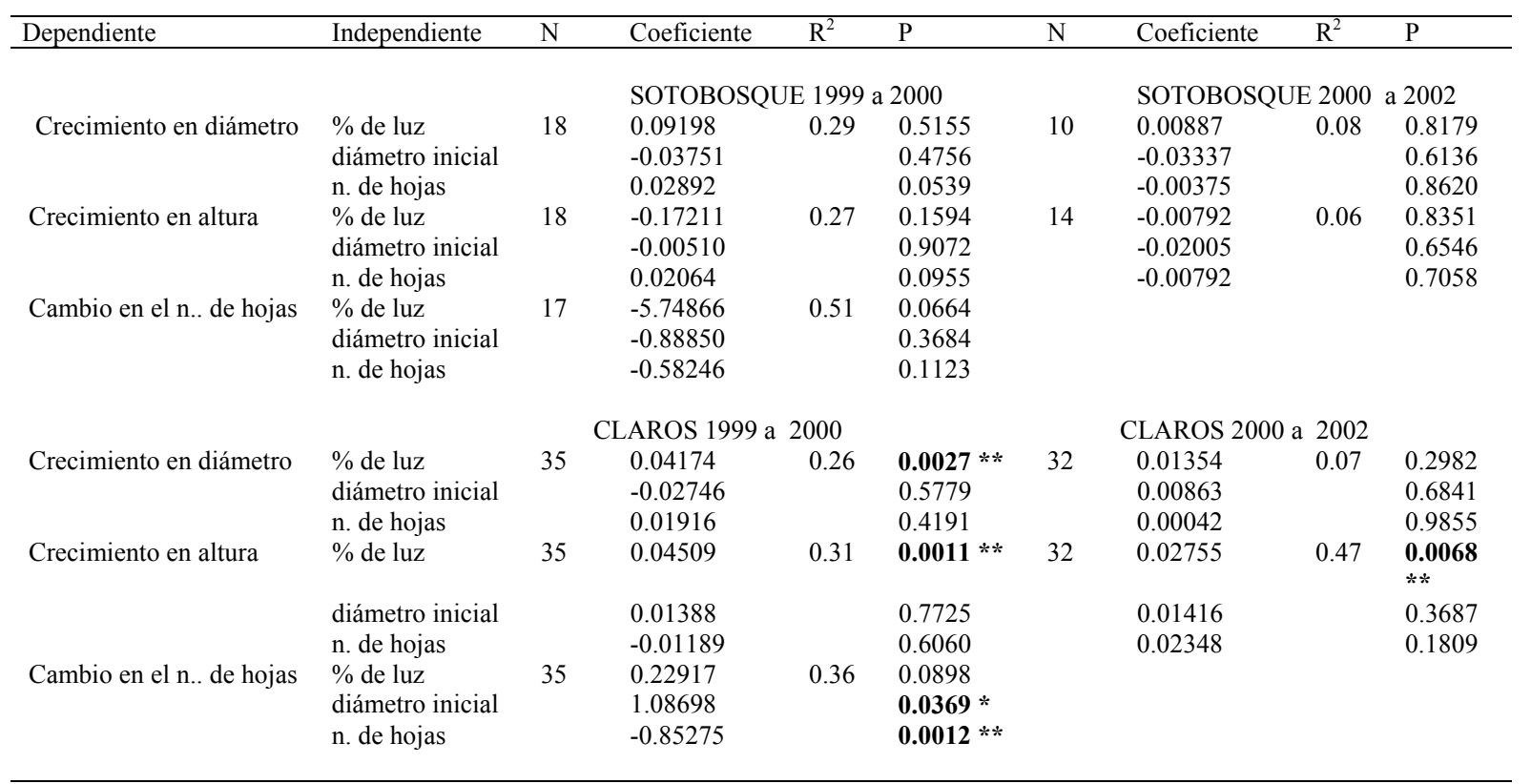

$\mathrm{P}=0.82$ ). Comparativamente con las plantas en ambientes naturales o en claros, es notable el diámetro y altura alcanzados por las plantas en estos ambientes de niveles de luz altos: en la plantación pura en bajío luego de dos años, el diámetro fue cercano a $5 \mathrm{~cm}$ $(\chi \pm$ DS: $47 \pm 16 \mathrm{~mm})$ y la altura de casi $5 \mathrm{~m}$ $(\chi \pm \mathrm{DE}: 5129 \pm 1985 \mathrm{~mm}, \mathrm{n}=17)$. En la plantación mixta luego de tres años el diámetro y la altura fueron comparativamente menores (diámetro $\chi+\mathrm{DE}: 13+5 \mathrm{~mm}$; altura $897 \pm 495 n=34$ ). En la Figura 4 se muestra la relación alométrica de los individuos sembrados.

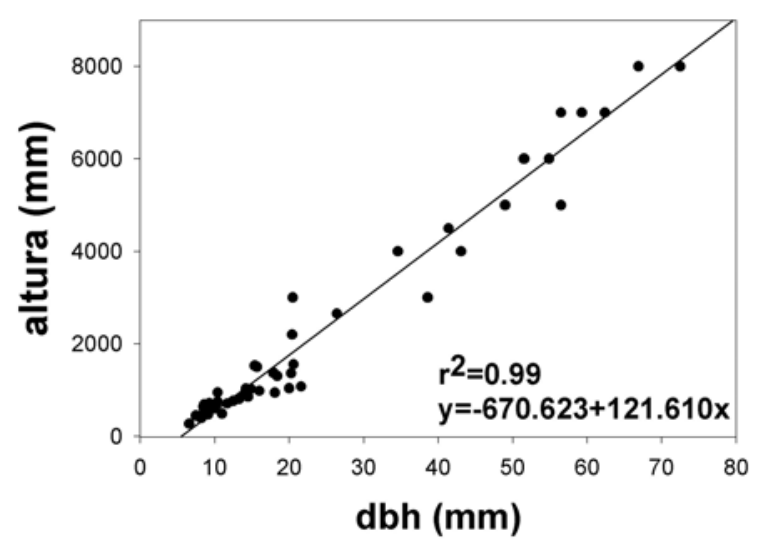

Figura 4. Relación alométrica de los individuos de Dipteryx micrantha de las plantaciones puras y mixtas.

\section{Discusión}

Dipteryx panamensis, una especie congenérica muy estudiada en Centro América y similar ecológicamente a $D$. micrantha, ha sido clasificada como una especie que requiere de luz como adulto. Sin embargo en sus estadíos jóvenes, las especies de bosque primario en general, son extremadamente tolerantes a la sombra (Fetcher et al.,1996). En efecto, casi todas las especies de árboles del dosel parecen ser tolerantes a la sombra en cierto grado (Clark \& Clark, 1984; Clark \& Clark, 1987), por lo que los conceptos de dependencia de claros y necesidades de la regeneración requieren ser redefinidos.

Los claros permiten un incremento en los niveles de luz y consecuentemente el crecimiento de las plantas pero al "cerrarse" en unos años, se retorna a niveles de luz similares a los del sotobosque, como hemos visto en el presente trabajo y otros (De Steven, 1988; Brokaw \& Whitmore, 1992; Van der Meer, 1998; Fraver et al., 1998). Por esto, se postula que las plántulas deberían estar presentes cuando se crea el claro para tener alguna ventaja de tamaño y mejor oportunidad de supervivencia y crecimiento. Esto tal vez explica la diferencia de nuestros resultados con aquellos de Cintra \& Horna (1997) que transplantaron 96 plántulas recién germinadas a claros donde sobrevivieron el $35 \%$, mientras que si son transplantadas luego de un año, como en este trabajo, pueden sobrevivir el 85\%. Aunque los claros son lugares ventajosos para el crecimiento, la caída de árboles o creación de claros, es por lo general un 
evento no muy común. Por ejemplo, en Cocha Cashu el promedio de la mortalidad de árboles es de $1.6 \%$ (Gentry \& Terborgh, 1990).

Con relación al efecto de luz en el crecimiento se observa que este depende de los rangos de luz estudiados: en los ambientes muy oscuros como el sotobosque ( $<5 \%$ luz) y en los ambientes donde la luz no es limitante como en las plantaciones puras o mixtas $(>20 \%)$, la luz no es determinante en el crecimiento o posiblemente no es el factor más importante que determina el crecimiento. Sólo en los claros donde los niveles de luz son intermedios (aprox. 6-20\%) se observa una relación directa entre la luz y el crecimiento.

Cuando la luz no es determinante en el crecimiento hacia arriba, otros factores como calidades intrínsecas de las semillas o plántulas o la presencia de agua entre otros, podría ser más determinante. Este último factor ha mostrado resultados diferentes en diversos estudios. Comparaciones de la disponibilidad de agua en claros y sotobosque han mostrado más, menos o similar disponibilidad. Sin embargo es menos claro cómo esto se traduce en el estrés hídrico de la planta (Poorter \& Hayashida, 2000). Veenendaal et al. (1995) encontraron más estrés hídrico en plántulas del sotobosque que en plántulas en claros sometidas a diferentes regímenes de irrigación.

Aunque este estudio no ha medido el factor agua, se sugiere que este factor, podría ser la razón para el gran crecimiento observado en las plantaciones puras en bajío (o planicie de inundación) ya que mucho del incremento en biomasa de hojas y altura para plántulas de otras tres especies de árboles estudiadas por Poorter \& Hayashida (2000), ocurre durante la época de lluvia. Para D. panamensis en particular la mayor foliación ocurre durante la primera mitad de la época de lluvia (Wright \& VanShaik, 1994). Para el caso de las plantas en plantaciones, el hecho de haber sido sembradas a comienzos de la época de lluvia puede también haber conferido una ventaja.

La calidad del suelo, un factor que también puede haber influido en el crecimiento, no fue caracterizada en este estudio. Tampoco se estudio el crecimiento bajo suelo en estas especies aunque existen estudios de la relación del crecimiento de la raíz con la luz, para otras especies congenéricas. Fetcher (1983) no encontró un efecto en el crecimiento en plántulas de D. panamensis transplantadas de sombra total o parcial a ambientes de luz total, como podrían ser las plantaciones, pero sí un incremento en el peso seco debido a una tasa más alta de la proporción raíz:tallo, es decir una mayor inversión en raíz. Una planta con raíz más profunda o sana, aunque no necesariamente sea alta, probablemente tiene ventajas en sobrevivencia y crecimiento futuros.

El conocimiento de los factores que influyen en la regeneración temprana de una especie en ambientes naturales es útil para el manejo silvicultural. En el caso de $D$. micrantha se observa que sólo plantaciones en bajío tienen un óptimo crecimiento al menos en su fase temprana observada $(<60 \mathrm{~mm}$ diámetro $)$. Sin embargo, el crecimiento posterior a esta fase así como las cualidades de la madera obtenida cuando el crecimiento es más acelerado, no se ha estudiado. Algunos autores mencionan que, para el caso de algunas especies maderables, las cualidades de la madera en plantaciones no es adecuada.

Aunque plantaciones y proyectos de reforestación en áreas de selva baja son todavía pocos, se espera que mientras más necesidad haya de secuestro de carbono éstos aumentarán. Dipterx micrantha, una especie de madera de gran densidad, debería ser una buena candidata para plantaciones por el volumen de carbono que produce, aunque tal vez no desde el punto de vista maderable debido al largo tiempo que implicaría alcanzar un tamaño comercial. Estimaciones del stock de carbón en $D$. panamensis de $3.4 \mathrm{~cm}$ y $7.4 \mathrm{~cm}$ de diámetro dan 1.62 . y $9.13 \mathrm{~kg} \mathrm{de}$ carbón respectivamente (Lois et al., 2003). Sin embargo, comercialmente para fines maderables y a pesar de su crecimiento acelerado a temprana edad comparativamente con ambientes naturales, es probable que requiera de un tiempo muy largo para alcanzar diámetros comerciales. El crecimiento a mayor edad en plantaciones es un aspecto que se necesita estudiar.

\section{Conclusiones}

- El crecimiento de plantas de las especies del género Dipteryx en claros es mayor que bajo sombra de la copa de los árboles.

- Los altos niveles de luz en los claros solo perduran por un par años antes de regresar a niveles de luz cercanos a los del sotobosque.

- La luz influye en el crecimiento de Dipteryx micrantha sólo a niveles intermedios como en los claros; a niveles muy bajos de luz como en el sotobosque o a niveles muy altos como en plantaciones, otros factores parecen tener mas relevancia.

- El crecimiento de Dipteryx en plantaciones puras en zonas inundables es extraordinariamente mayor que el registrado en plantaciones mixtas.

- Especies del género Dipteryx serían buenas para proyectos de secuestro de carbono.

\section{Agradecimientos}

Mi agradecimiento al Departamento de Biología de la Universidad de Turku donde analicé parte de los datos mostrados y a la Fundación de la Universidad de Turku que financió parte del trabajo de campo. A Gilbert Martinez quien me asistió en el campo con mucha dedicación. Al Sr. Demetrio Bedregal quien amablemente me mostró sus plantaciones y me permitió hacer las mediciones necesarias. Al Dr. Carlos Reynel por revisar mi manuscrito. 


\section{Literatura citada}

Brown N. 1993. The implications of climate and gap microclimate for seedling growth conditions in a Bornean lowland forest. Journal of Tropical. Ecology. 9: 153-168.

Brown N.D. \& Whitmore T.C. 1992. Do dipterocarp seedlings really partition tropical rain forest gaps? Philosophical Transactions of the Royal Society of London. Series B. : 335, 369-378.

Canham C.D. 1989. Different responses to gaps among shade-tolerant tree species. Ecology. 70: 548-550.

Chazdon R.L. \& Fetcher N. 1984. Phosynthetic light environments in a lowland tropical rain forest in Costa Rica. Journal of Ecology. 72: 553-564.

Chazdon R.L. 1988. Sunfleck and their importance in forest understory light. Advances on Ecological. Research. 18: $2-54$.

Cintra R. \& Horna V. 1997. Seed and seedling survival of the palm Astrocaryum murumuru and the legume tree Dipteryx micrantha in gaps in Amazonian forest. Journal of Tropical Ecology. 13: 257-277.

Clark D.A. \& Clark D.B. 1984. Spacing dynamics of a tropical rain forest tree: evaluation of the JanzenConnell model. American Naturalist. 124(6): 769-788.

Clark D.A. \& Clark D.B. 1992. Life history diversity of canopy and emergent trees in a neotropical rain forest. Ecological Monograph. 62(3): 315-344.

Clark D.B. \& Clark D.A. 1987. Population ecology and microhabitat distribution of Dipteryx panamensis, a neotropical rain forest emergent tree. Biotropica. 19(3): 236-244.

Clark D.B., Clark D.A. \& Rich P.M. 1993. Comparative analysis of microhabitat utilization by saplings of nine tree species in neotropical rain forest. Biotropica. 25(4): 397-407.

Connell J.H. \& Green P.T. 2000. Seedling Dynamics over thirty-two years in a tropical rainforest tree. Ecology. 81: $568-584$.

Dalling J.W., Lovelock C.E. \& Hubbell S.P. 1999. Growth responses of seedlings of two neotropical pioneer species to simulated forest gap environments. Journal of Tropical Ecology. 15: 827-839.

Delissio L.J., Primack R.B., Hall P. \& Lee H.S. 2002. A decade of canopy-tree seedling survival and growth in two Bornean rain forests: persistence and recovery from suppression. Journal of Tropical. Ecology. 18: 645-658.

Denslow J.S., Ellison A. \& Sanford R. 1998. Tree fall gap size effects on above and below ground processes in a tropical wet forest. Journal of Tropical Ecology. 86: 597-609.

De Steven D. 1988. Light gaps and long-term seedling performance of a Neotropical canopy tree (Dipteryx panamensis, leguminosae). Journal of Tropical Ecology. 4: 407-411.

Fetcher N., Strain B.R. \& Oberbauer S.F. 1983. Effect of light regimes on growth, leaf morphology and water relations of seedlings of Heliocarpus and Dipteryx. Oecologia. 58: 314-319.

Fetcher N., Haines B.L., Cordero R.A., Lodge D.J., Walker L.R., Fernandez D.S. \& Lawrence W.T. 1996. Responses of tropical plants to nutrients and light on a landslide in Puerto Rico. Journal of Ecology. 84: 331341 .
Foster R. 1990. Long-term change in the successional forest community of the Rio Manu Floodplain. In: A.H. Gentry ed. Four neotropical rainforests. Yale Univ. Press, New Haven, CT. : 565-572.

Fraver S., Brokaw N. \& Smith A. 1998. Delimiting the gap phase in the growth cycle of a Panamanian forest. Journal of Tropical Ecology. 14: 673-681.

Gentry A.H. \& Terborgh J. 1990. Composition and dynamics of the Cocha Cashu mature floodplain forest. In: In: A.H. Gentry ed. Four neotropical rainforests.. Yale Univ. Press, New Haven, CT. : 542-564.

Hubbell S.P. 1998. The maintenance of diversity in a neotropical tree community: conceptual issues, current evidence, and challenges ahead. In: F. Dallmeier \& J.A. Comiskey eds. Forest Biodiversity: Research, Monitoring and Modeling. UNESCO, Paris, France. : 17-44.

Hubbell S.P. \& Foster R.B. 1987. The spatial context of regeneration in a neotropical forest. In: A.J. Gray, M.J. Crawley and P.J. Edwards eds. Colonization, succession and stability. Blackwell Scientific Publications, Oxford.

Janson C.H. \& Emmons L.H. 1990. Ecological structure of the non-flying mammal community at Cocha Cashu Biological Station, Manu National Park, Peru. In: A.H. Gentry ed. Four Neotropical Rain Forest. Yale Univ. Press, New Haven, CT. : 314-338.

Lois C.J., Siccama T.G., Condit R. \& Morales J. 2003. Analysis of alternative methods for estimating carbon stock in young tropical plantations. Forest Ecology and Management. 184: 355-368.

Osunkoya O.O., Ash J.E., Graham A.W. \& Hopkins M.S. 1993. Growth of tree seedlings in tropical rain forest of North Queensland. Australia. Journal of Tropical Ecology. 9: 1-19.

Osunkoya O.O., Ash J.E., Hopkins M.S. \& Graham A.W. 1994. Influence of seed size and seedling ecological attributes on shade-tolerance of rain-forest tree species in northern Queensland. Journal of Ecology. 82: 149163.

Poorter L. \& Hayashida-Oliver Y. 2000. Effects of seasonal drought on gap and understorey seedlings in a Bolivian moist forest. Journal of Tropical Ecology. 16: 481-498.

Romo M., Ruokolainen K. \& Rajaniemi S. 2004. Population structure and recruitment of an emergent tree, Dipteryx micrantha in different habitats of a Peruvian floodplain forest. In: Regeneration ecology and population structure of the emergent tree Dipteryx micrantha (Fabaceae) in floodplain forest of the Manu River, Amazonia Peru. Ann. Univ. Turkuensis Sarja-Ser AII- Tom 180, Chapter IV.

Smith A., Hogan K. \& Idol J. 1992. Spatial and temporal patterns of light and canopy Structure in a lowland tropical moist forest. Biotropica. 24: 503-511

Van der Meer P.J., Sterk F.J. \& Bongers F. 1998. Tree seedlings performance in canopy gaps in a tropical rain forest at Nourges, French Guyana. Journal of Tropical Ecology. 4: 119-137.

Veenendaal E.M., Swaine M.D., Agyeman V.K., Blay D., Abebrese B.K. \& Mullins C.E., 1995. Differences in plant and soil water relationships in and around gap in West Africa during the dry season may influence seedling establishment. Journal of Tropical Ecology. 83: 83-90. 
Volpato E., Schmidt P. \& Araujo V. 1973. Situaçăo dos plantios experimentais na reserva forestal Ducke. Acta Amazonica. 3: 71-82.

Wright S.J. \& Van Shaik C.P. 1994. Light and phenology of tropical trees. American Naturalist. 143: 192-199.
Zagt R.J. 1997. Tree demography in the tropical rain forest of Guyana. Tropenbos Guyana Series. Vol. 3. $251 \mathrm{pp}$. ${ }^{1}$ University of Turku, Department of Biology, Turku 20014. Finland. Dirección actual: Av. Pérez Aranibar
1730, Lima 27, Perú. 